\title{
Lutte contre la Covid-19 : une bonne raclée contre les droits humains en République Démocratique du Congo
}

\author{
Par KALALA ILUNGA MULUMBA Matthias*, KALENGA NGOY Marie \\ Thérèse ${ }^{* *}$, KIMPESA YA MULENDA***
}

\section{INTRODUCTION}

Dans sa dissertation doctorale Kalala Ilunga Mulumba Matthias affirmait il y a peu que : « depuis son accession à l'indépendance, il y a six décennies, la République Démocratique du Congo est confrontée aux problèmes multiples et de tout genre. Au nombre de ceux-ci, se rangent en ordre utile l'insécurité, la violation des droits de l'homme, les maladies épidémiques et endémiques, le manque d'eau potable et de l'électricité, des routes, des hôpitaux; l'analphabétisme, le tribalisme, la corruption, la pauvreté, etc. » ${ }^{1}$. Comme si cela ne suffisait pas, il faudra ajouter à ce lot de souffrances, l'apparition de ce monstre ennemi contre lequel tous les Etats se battent, le corona virus 2019. Quel miracle la République Démocratique du Congo devra-t-elle opérer pour protéger sa population?

En effet, la satisfaction des besoins collectifs de la population étant l'une des missions essentielles de tout Etat, les gouvernants de la République Démocratique du Congo devront inéluctablement s'appuyer sur les services publics. La notion de service public elle-même, n'est concevable qu'en rapport avec la mission de satisfaction de l'intérêt général. Voilà pourquoi, affirment J.F. Lachaume et autres que les ouvrages de Droit administratif contiennent des développements plus au moins substantiels sur les services publics, ce qui n'est pas moins surprenant puisque l'organisation et la gestion des services publics en vue de la satisfaction de l'intérêt général, par l'octroi des prestations de toute nature, constituent la responsabilité des personnes publiques. ${ }^{2}$

Cependant, dans l'une de ses études, l'auteur précité concluait fatalement que les services publics congolais ne concourraient presque véritablement pas à la satisfaction de l'intérêt général ${ }^{3}$. Devant cette situation, quels devraient être les stratégies à mettre en place pour affronter ce monstre de coronavirus 2019 qui n'épargne aucune nation? Pourtant, le

* Docteur en Droit, Université de Kamina, Institut Supérieur des Techniques Médicales de Lubumbashi, Université Protestante de Lubumbashi R.D. Congo, courriel : kalalamatthias@yahoo.fr

** Assistante à l'Institut Supérieur des Techniques Médicales de Lubumbashi, R.D. Congo, courriel : mtkalenga@gmail.com

*** Assistant à l'Institut Supérieur des Techniques Médicales de Lubumbashi, R.D. Congo.

1 Kalala Ilunga Mulumba Matthias, Gestion des services publics et protection des droits humains, thèse de doctorat, Université de Lubumbashi, Lubumbashi RD Congo, 2019, inédite, P.1.

2 Lachaume, J.F., Boiteau, \& Pauliat, H., Droit des services publics, éd. $3^{\text {ème }}$ éd. Coll. Armand Colin, éd. Dalloz, Paris, 2004, p. 7.

3 Kalala Ilunga Mulumba Matthias, Op. cit. p.3. 
fondement de la notion du service public tel que la République Démocratique du Congo l'a hérité de la colonisation, à savoir l'intérêt général, est encore une notion vague aussi bien dans le chef des agents publics que dans celui des usagers des services publics. Cet état de chose serait, en grande partie, due au fait que la notion même de l'Etat promoteur des services publics est aussi vague et mal intériorisée par les Congolais.

C'est ce qui serait, d'ailleurs, à la base de tant de dysfonctionnements de la plupart des services publics, leur mauvaise gestion entraînant, par ce fait même, l'insatisfaction des besoins collectifs des Congolais et la violation fréquente des droits et libertés fondamentales ${ }^{4}$. Corrélativement, l'Etat - les services publics - l'intérêt général - les droits humains sont tellement imbriqués qu'il est souvent très difficile de les dissocier. Eh bien, si l'Etat doit assurer le respect des droits de l'homme et la satisfaction de ses besoins, celan'est possible que s'il a des services publics compétitifs, efficaces, efficients, performants et émergents ${ }^{5}$.

En ce qui concerne les droits de l'homme, précisons que ceux-ci relèvent du Droit naturel et, par conséquent, considérés comme préexistant à toute société humaine, une société politiquement organisée, c'est-à-dire, l'Etat lui-même. Les pères de cette conception, parmi lesquels, on citera Grotius, ont une conviction que, tout humain (individu) possède un ensemble des prérogatives ou facultés inhérentes à sa nature qu'on ne peut méconnaitre sans atteinte à celles-ci. ${ }^{6}$ Pris sous cet angle, les droits de l'homme sont à entendre dans cette étude comme ce chapelet des garanties et besoins fondamentaux de l'homme qu'il faudrait promouvoir et satisfaire.

A titre illustratif, si l'on soutient que l'homme a droit à la vie, à l'éducation, à la santé, il s'agit là des besoins qu'il faut satisfaire, par et à travers, la création de l'armée, la police, les Cours et tribunaux, les hôpitaux, etc. Chacun de ces services devant veiller dans la mesure du possible à ce que rien ne mette fin à une vie humaine. Il se crée ainsi une relation profonde, pour ne pas dire un mariage entre les droits humains et les services publics appelés constamment à en assurer le respect, la promotion et la protection.

Les violations des droits régulièrement décriés en République Démocratique du Congo sont souvent ou, sinon fréquemment dues à la faiblesse ou à l'inefficacité des services publics appelés à en assurer la protection. Au regard de ce qui précède, cette étude tentera, tant soi peu, de proposer des pistes de solutions idoines en vue d'assurer une maximale protection de la population congolaise contre cette pandémie à coronavirus 2019 .

Pour y parvenir, le recours aux méthodes exégétique et dialectique appuyées par les techniques documentaire et d'interview pourront nous être utiles dans cette étude. Aussi gravitera-t-elle autours des trois axes principaux, à savoir : des actions gouvernementales de lutte contre la covid-19 attentatoires contre les droits humains, la nécessaire protection des droits humains dans la lutte contre la covid-19 et les stratégies de lutte contre la covid-19 dans le respect des droits humains.

4 Idem, p.8.

5 Idem, p.9.

6 OBERDORFF Henri, Droits de l'homme et libertés fondamentales, L.G.D.J., Paris, 2008, p. 42. 


\section{A. DES ACTIONS GOUVERNEMENTALES DE LUTTE CONTRE LA COVID-19 ATTENTATOIRES AUX DROITS HUMAINS}

\section{Légalité et légitimité des mesures gouvernementales contre la covid-19}

La pandémie à coronavirus 2019 n'est pas moins une guerre qui demandent que des mesures d'urgence soient prises et mises en place pour la combattre. Malheureusement, il se fait que l'ensemble de toutes ces mesures est un coup dur porté contre les droits humains. De la proclamation de l'état d'urgence sanitaire jusqu'à l'ensemble des mesures de prévention et de prises en charges des personnes touchées par ce monstre de maladie, tout est attentatoire aux droits et libertés fondamentales des citoyens. Il en est de même de l'inaction qui elle aussi serait pire que les atteintes portées contre les droits humains.

Les Etats n'ont donc pas de choix, ils doivent agir mais en essayant de concilier lutte contre covid-19 et respect des droits humains. Ce qui constitue une tâche ardue et un énorme défi pour la plupart des Etats du monde. Comment alors les Etats devront y faire face sans se compromettre? Il nous semble, à notre humble avis, qu'ils devront, à tout point de vue, se placer du côté de la légalité pour pouvoir légitimer et justifier tous leurs moyens d'action. Toutes actions gouvernementales étant reconnues attentatoires aux droits humains, celles-ci devraient être pratiquées avec discernement pour éviter tout dérapage ou anarchie, d'où la nécessité de l'instauration des régimes d'état d'urgence afin de permettre aux gouvernants de légaliser leurs actions et de prendre toutes les mesures idoines pour lutter efficacement contre la pandémie.

En effet, l'instauration des régimes d'état d'urgence et les mesures d'exception adoptées par la plupart des Etats, en général, et la République Démocratique du Congo, en particulier, en première réponse pour prévenir et/ou endiguer la propagation de la pandémie du Covid-19 ont été, dans la grande majorité, conformes au constitutionnalisme et à l'État de droit tant du point de vue procédural (proclamation, consultation et/ou intervention d'organes constitutionnels tels que les parlements et les cours constitutionnelles, limitation dans le temps, etc.) que sur le fond (respect des dispositions constitutionnelles, des cadres juridiques existants sur les plans national, régional et continental, mais aussi des conventions internationales et régionales pertinentes en matière de droits humains « pactes internationaux relatifs respectivement aux droits civils et politiques, et aux droits économiques, sociaux et culturels...; Charte africaine des droits de l'Homme et des peuples... », qui sont autant d'instruments posant des balises strictes pour la mise en œuvre et le déploiement de telles mesures d'exception). Ces dispositions ont été justifiées par la priorité donnée au droit à la santé.

Elles ont été de différents ordres mais sont relativement similaires ${ }^{7}$ : déclarations d'état d'urgence; couvre-feux; fumigation de masse et désinfection des espaces publics, des rues, des marchés ainsi que des gares ferroviaires et des stations d'autobus et de taxi; imposition

7 Rapport analytique du webinaire, 23 avril 2020, l'impact de la crise du Covid-19 sur le constitutionnalisme et l'État de droit dans les pays francophones et lusophones d'Afrique de l'Ouest et 
de la distanciation sociale; confinement partiel; soutien aux groupes les plus vulnérables; restrictions sur les rassemblements sociaux / publics (y compris dans les églises et les mosquées); circulation restreinte de tous les transports en commun; fermeture de toutes les structures scolaires et de formation; fermeture des services publics, commerces, restaurants et autres installations collectives à l'exception des services essentiels; encouragement à une hygiène rigoureuse (par exemple, se laver fréquemment les mains, etc.); interdictions de voyager et restrictions à la libre circulation des personnes dans le pays et à l'étranger; fermeture des frontières (terrestres, aériennes, maritimes); etc.

\section{Mesures gouvernementales et obligation du respect des droits humains}

En effet, Amnisty International a, dans les premières heures qui ont suivi la reconnaissance de la maladie à coronavirus 2019 comme une pandémie, demandé à tous les gouvernements et autres acteurs concernés par la flambée de maladie à coronavirus 2019 (Covid-19) de veiller à placer le droit international relatif aux droits humains et les normes en la matière au cœur de l'ensemble des mesures prises pour lutter contre le virus, afin de protéger au mieux la santé publique et de venir en aide aux personnes qui risquent davantage d'en subir les conséquences néfastes ${ }^{8}$.

Ce document de Amnisty International ${ }^{9}$ examine dans un premier temps les préoccupations relatives aux droits humains qui, bien souvent, se posent aux différentes étapes d'intervention des États en réponse à une épidémie - avec des références précises aux mesures prises par les États face à la covid-19. Il synthétise dans un deuxième temps les obligations qui leur incombent en matière de droits humains, ainsi que les principaux textes, normes et principes dans ce domaine qui doivent transparaître dans leur action de lutte contre le nouveau coronavirus. Sont également traitées les considérations relatives aux droits humains qui sont en jeu dès lors que les États imposent des mesures préventives de protection de la santé publique, telles que la mise en quarantaine et l'interdiction de se déplacer, ainsi que les obligations qui sont les leurs, à savoir garantir l'accès à des soins, des biens et des services de prévention; veiller à l'accessibilité physique et financière des soins; garantir la sécurité sociale et les droits des travailleurs et travailleuses; prévenir la stigmatisation et la discrimination; et protéger le personnel de santé. Le document aborde enfin les obligations des États en matière d'aide et de coopération à l'échelon international, ainsi que d'appui au rétablissement à long terme et d'action à mener dans le prolongement de l'épidémie ${ }^{10}$.

d'Afrique Centrale, Institute international pour la démocratie et l'assistance électorale, disponible sur http://www.idea.int consulté le 15/12/2020.

8 Amnisty International, Mesures prises face à la covid-19 et obligations des états en matière de droits humains : observations préliminaires, Déclaration publique du 12/03/2020, disponible sur www.amnesty.org.

9 Ibidem.

10 Idem, p.2. 
Dans le souci de protéger au mieux la santé publique et de venir en aide aux personnes et groupes de personnes qui sont les plus vulnérables, il faut dès le départ placer les droits humains au cœur de toutes les mesures qui sont prises dans les domaines du traitement, de l'endiguement, de la préparation et de la prévention. Or, nombre des initiatives visant à limiter la propagation de la COVID-19 semblent avoir été proposées et mises en œuvre sans qu'aient été pleinement prises en compte leurs éventuelles conséquences sur la jouissance de l'ensemble des droits humains par toutes et tous, les personnes infectées comme celles qui ne le sont pas, ainsi que les personnes à risque comme celles qui n'en font pas partie ${ }^{11}$.

Les États ont notamment pour obligation de garantir les droits civils, culturels, économiques, politiques et sociaux, conformément au principe d'interdépendance et d'indivisibilité des droits humains. Le plein respect de ces droits est fondamental à toutes les étapes de la crise; il convient en conséquence de ne pas le juger comme un luxe qu'on ne peut se permettre qu'une fois la menace pour la santé publique réduite. Les personnes touchées par une situation d'urgence pour la santé publique, y compris celles qui sont déplacées en raison d'une situation de ce type, conservent le droit de bénéficier de la protection pleine et effective du droit relatif aux droits humains.

\section{Les mesures d'urgence attentatoires aux droits humains pour la réduction de l'exposition à la covid-19}

Comme on le sait, l'action administrative se présente généralement sous deux formes, à savoir : la police administrative et le service public. En effet, toutes les mesures de police administrative constituent une menace pour les libertés individuelles. En conséquence, il sied qu'elles soient limitées et contrôlées. Le pouvoir de police ne peut porter atteinte aux libertés publiques que dans la mesure où cela est absolument nécessaire.

En République Démocratique du Congo, ces mesures ont été de différents ordres mais actuellement celles qui subsistent réellement et qui, au dire de beaucoup d'analystes violent les droits humains sont les suivants : couvre-feux; imposition de la distanciation sociale même si c'est souvent sans résultats attendus; restrictions sur les rassemblements sociaux / publics; encouragement à une hygiène (par exemple, se laver fréquemment les mains, etc.); fermeture de nouveau de toutes les structures scolaires et de formation; Etc.

De toutes ces mesures, nous allons tenter d'analyser quelques-unes dont :

\section{Mise en quarantaine}

La mise en quarantaine (qui consiste à isoler les personnes qui sont susceptibles d'avoir été exposées à une maladie infectieuse ou qui en présentent les symptômes) est une mesure couramment appliquée par les pays pour enrayer la progression de maladies infectieuses, la covid-19 ne faisant pas exception. Selon les informations dont on dispose, des mesures plus 
ou moins drastiques de quarantaine sont imposées aujourd'hui, couvrant parfois des villes et des régions entières ${ }^{12}$. Ces mesures ont une incidence sur le droit de circuler librement des personnes et, selon la façon dont elles sont appliquées, peuvent aussi s'apparenter à une privation arbitraire de liberté.

La mise en quarantaine ou à l'isolement peut conduire à des mesures coercitives ou à des situations dans lesquelles les besoins fondamentaux des personnes placées en quarantaine ne sont pas satisfaits. Des femmes, des enfants et des adolescents pourront se retrouver piégées dans leurs foyers et confrontés à des violences interpersonnelles sans possibilité de s'échapper ${ }^{13}$.

Des informations font également état de personnes qui se heurtent à des obstacles supplémentaires pour exercer leurs droits humains parce qu'elles sont placées en quarantaine, y compris pour se procurer des produits de première nécessité (nourriture et produits d'hygiène, notamment) et pour se faire soigner, et qui voient leur emploi et leur salaire mis en péril parce qu'elles sont dans l'impossibilité d'aller travailler. Une mesure de quarantaine entraîne d'autres conséquences sur le plan des droits humains : elle peut avoir un effet disproportionné sur les personnes pauvres, qui n'ont peut-être pas les moyens d'acheter entre autres des produits alimentaires et de santé. Il est également possible qu'elles n'aient pas suffisamment d'économies pour tenir pendant une période de congés sans solde. Ce type de mesure n'est autorisé par le droit international relatif aux droits humains que dans des circonstances limitées.

En République Démocratique du Congo, l'application de cette mesure pose d'énormes problèmes à la population qui ne vit, du jour au lendemain, que de l'économie informelle. Avec son économie extravertie, tributaire du secteur minier, et fortement dépendante des intrants et des produits de première nécessité et des denrées de base importés, la République Démocratique du Congo devrait voir sa croissance économique baisser sensiblement et ses comptes macroéconomiques se détériorer en profondeur si une riposte d'envergure n'est pas envisagée. Le secteur extractif qui constitue le principal levier de sa croissance participe à plus ou moins $30 \%$ dans ses recettes budgétaires. S'agissant des recettes douanières et d'assises sur le commerce extérieur du pays, elles participent à près de $40 \%$ dans le budget en recettes ${ }^{14}$.

La République Démocratique du Congo est l'un des pays africains pouvant être le plus secoué par les effets du Covid-19. En effet, sur huit facteurs de risque, six pèsent sur elle et sur ses perspectives de développement, à savoir l'exposition aux contacts internationaux, l'efficacité du système de santé, la population totale dans les zones urbaines,

12 Business Insider, cite par Amnisty international, Op. cit. p.5.

13 Fons mondial, Note d'orientation : les droits humains en temps de pandémie de covid-19, 14 avril 2020, p.2.

14 République Démocratique du Congo, Gouvernement, Impacts sanitaires et socio-économiques de la covid-19 en République Démocratique du Congo, analyse prospective et orientations de la riposte multisectorielle, Kinshasa, Mai 2020, p.14. 
la transparence dans la gouvernance politico-administrative, l'ampleur des conflits armés et des problèmes sécuritaires, et l'importance des déplacements de la population sur le territoire national. La riposte du gouvernement au Covid-19 devrait en tenir compte pour cause de pertinence et d'efficacité dans l'action ${ }^{15}$.

\section{Interdiction et limitation des déplacements}

D'autres mesures adoptées par les États, telles que les interdictions et les limitations des déplacements, peuvent également avoir une incidence sur le droit de circuler librement, qui inclut la liberté de quitter n'importe quel pays et le droit de ne pas être arbitrairement privé du droit d'entrer dans son propre pays ${ }^{16}$. Plusieurs pays ont fermé une partie de leurs frontières, ou interdit les déplacements en provenance ou à destination des régions où sont recensés un grand nombre de cas de covid-19, ce qui a souvent des répercussions sur les personnes qui tentent de rejoindre leur domicile et leur famille, de mener à bien leur activité professionnelle, ou d'avoir accès à l'éducation à l'école et à l'université.

L'Organisation mondiale de la santé déconseille de façon générale d'appliquer des restrictions aux voyages ou aux échanges commerciaux vers les pays connaissant une flambée épidémique de covid-19, hormis dans des circonstances limitées, en raison des risques posés par cette approche. Selon les dernières recommandations en date de l'Organisation, « les restrictions peuvent interrompre l'aide et le soutien technique nécessaires, perturber l'activité économique, et avoir des retombées socioéconomiques négatives sur les pays touchés ».

\section{Soins, biens et services de prévention}

Le système de santé de la République Démocratique du Congo se trouve déjà aux prises avec une surcharge de travail à cause de l'épidémie d'Ebola, de la rougeole et celle du choléra ainsi que d'autres maladies endémiques comme le paludisme et n'est probablement pas à même de faire totalement face aux exigences et méfaits du Covid-19. Les services de l'Organisation mondiale de la santé appuient, toutefois avec le corps médical, pour assurer une riposte acceptable. Les mesures préventives prises par le gouvernement devraient être strictement appliquées mais avec un bon ciblage des actions de terrain, notamment par une mise en quarantaine des contaminés et un suivi des personnes à risque.

Le droit à la santé comprend les mesures nécessaires pour assurer la prophylaxie et le traitement des maladies épidémiques, endémiques, professionnelles et autres, ainsi que la lutte contre ces maladies. Dans le contexte d'une flambée épidémique, cela signifie que les États ont l'obligation de veiller à la disponibilité et à l'accessibilité pour toutes et tous de soins, de biens, de services et d'informations de prévention. Il s'agit notamment de

15 République Démocratique du Congo, Gouvernement, Impacts sanitaires et socio-économiques de la covid-19 op cit., p.15.

16 Amnisty International, Op. cit. p.4. 
communiquer des informations exactes, scientifiquement fondées et facilement accessibles sur la façon dont les gens peuvent se protéger, et de veiller à ce que les biens nécessaires à la prévention soient disponibles, physiquement et financièrement, pour toutes et tous.

Des pénuries de produits de protection, tels que des masques et des solutions hydroalcooliques, ont été signalées dans certaines régions. Une organisation non gouvernementale de Hong Kong, Society for Community Organisation, a par exemple, indiqué que près de $70 \%$ des familles à faible revenu du pays n'avaient pas les moyens d'acheter du matériel de protection, tel que des masques et des solutions hydroalcooliques ${ }^{17}$. Les États doivent veiller à ce que ces produits soient disponibles, à un coût abordable, dès lors que leur utilisation est recommandée.

\section{B. LA NECESSAIRE PROTECTION DES DROITS HUMAINS DANS LA LUTTE CONTRE LA COVID-19}

\section{Notions sur les droits humains}

Les droits de l'homme puisent, leur légitimité dans une certaine conception de l'homme c'est-à-dire dans des valeurs jugées fondamentales qui permettent de distinguer de manière décisive l'espèce humaine de l'espèce animale. On peut parler à juste titre d'un principe d'humanité. Les fondements philosophiques par fois religieux, ont permis l'adoption de nombreux textes reconnaissant des droits de l'homme et des libertés. Certains sont plus emblématiques que d'autres, c'est le cas de la déclaration des droits de l'homme du citoyen de $1789 .^{18}$

Sur un plan strictement linguistique, un débat existe sur les termes les plus adaptés à employer. Faut-il parler de droits de l'homme ou de droits humains. Si la France s'obstine à employer le terme de droits de l'homme, de nombreux autres pays parlent de plus en plus souvent des droits humains. Ainsi, la ministre des affaires étrangères de Suisse considèrent que «l'expression de droits de l'homme n'est plus adapté à la société moderne et égalitaire d'aujourd'hui, la langue se doit d'accompagner les évolutions sociales, et l'expression droits humains se comprend comme une adaptation logique à l'égalité de sexe ». ${ }^{19}$

Dans cet article, nous préférons utiliser, pour notre part, l'expression droits humains qui nous semble être l'expression la plus favorable en rapport avec l'évolution de mœurs, d'attitudes et de politique d'intégration. Cependant, nous ne nous en voudrons pas à ceux qui préfèrent volontiers continuer à être attaché à l'expression classique de droits de l'homme. Les libertés publiques appartiennent en propre à la sphère du Droit et se bornent à dire ce qui est. Elles se rattachent à un ordre juridique national. Elles sont donc reconnues dans le droit positif interne des Etats. Par conséquent, elles ne s'appliquent qu'à l'intérieur de leurs

17 Amnisty International, Op. cit., p.5.

18 OBERDORFF, Op.cit., p.21.

19 MORANGE, J., Libertés publiques, éd. PUF, Paris 2007, p.10. 
frontières étatiques aux personnes qui vivent sur leur territoire. Elles peuvent se développer et s'épanouir si les lois internes les admettent et les organisent.

Les Etats peuvent aussi, s'ils le souhaitent, réduire ou limiter l'exercice de ces libertés en fonction, par exemple, de leur conception ou des nécessités de l'ordre public. Cette réduction est parfois rendue indispensable pour sauvegarder la démocratie, notamment en cas de circonstances exceptionnelles. Ainsi, pour beaucoup d'auteurs, si les droits de l'homme relèvent surtout d'une philosophie, les libertés publiques; au contraire, sont strictement de l'ordre du Droit et, donc, plutôt déterminées par le législateur.

Les différences ne sont pas pour autant si catégoriques. En réalité, les droits de l'homme sont pleinement intégrés dans une perspective juridique. Ils ne relèvent pas d'une rêverie propre à des juristes égarés, mais, sont dotés d'un statut juridique qui les transforme en une catégorie juridique à laquelle est attaché un statut protecteur. Ils relèvent, plus souvent qu'on ne le croit, de l'ordre du Droit et non de la simple philosophie. Le fait que ces droits constituent désormais une catégorie juridique est le signe d'une profonde mutation significative d'un passage d'une idéologie au droit.

Cette évolution permet de donner une définition des droits d l'homme : « des prérogatives gouvernées par des règles, que la personne détient en propre dans ses relations avec les particuliers et le pouvoir $» .^{20}$ Partis d'une ambiance philosophique marquée, à la fin du XVIII ${ }^{\text {ème }}$ siècle, par l'école du droit naturel, les droits de l'homme se sont largement juridicisés pour participer à la concrétisation d'un Droit mondial opposable devant des juridictions.

Le recours à la notion de droits fondamentaux est largement inspiré par la doctrine constitutionnelle allemande. En effet, le titre premier de la loi fondamentale de la République Fédérale d'Allemagne du 23 Mai 1949 est justement consacré aux droits fondamentaux. L'article $1^{\text {er }}$ en fixe la problématique de manière très claire : l'intangibilité de la dignité de l'être humain, des droits inviolables et sacrés pour l'être humain, les obligations pesant sur les pouvoirs exécutif, législatif et judiciaire de le respecter comme droits directement applicables. La loi fondamentale dresse ainsi une théorie juridique des droits fondamentaux.

De nombreux textes internationaux ont adopté cette référence aux droits fondamentaux. A titre illustratif, la Déclaration Universelle des Droits de l'Homme de 1948 le fait dans l'un de ses considérants : considérant que les Etats membres se sont engagés à assurer, en coopération avec l'organisation des Nations Unies, le respect universel et effectif des droits de l'homme et des libertés fondamentales.

De manière traditionnelle, il est possible de distinguer des générations des droits de l'homme en fonction du moment où ils sont apparus :

- Une première génération avec les droits civiques et politiques au XIII ${ }^{\text {ème }} \mathrm{XIX}^{\text {ème }}$ siècles,

- Une deuxième génération avec les droits économiques et sociaux au XX ${ }^{\text {ème }}$ siècle,

- Une troisième génération des droits de l'homme à la fin du XX ${ }^{\text {ème }}$ et auXXI ${ }^{\text {ème }}$ siècle.

20 MOURGEON, J., Les droits de l'homme, coll. Que sais-je?, 8éme éd. PUF, Paris, 2003, p.8. 
La troisième génération connaît actuellement, dans plusieurs domaines, une inflation des nouveaux droits de l'homme. Le terme d'inflation est ici volontairement utilisé, car il permet de mieux comprendre le recours très fréquent à cette notion des droits de l'homme par une forme d'abus de langage. Comme en matière monétaire, cette inflation a des effets négatifs sur le contenu et la protection de ces nouveaux droits ${ }^{21}$. Ces nouveaux droits concernent d'abord les évolutions sociétales comme :

- Les droits de l'environnement (droit à un air pur, droit à un environnement sain, la protection contre les bruits, etc.),

- Les droits de la santé (droit des malades, droit à la santé...),

- Le droit de la sécurité,

- Le droit au transport,

- Le droit au logement,

- Le droit au (ou du) développement durable,

- Le droit à la différence,

- Etc.

Ils concernent ensuite les évolutions technologiques :

- Les droits des communications électroniques (droits ou libertés de la vie privée face à l'informatique, le secret des correspondances par voie de télécommunication...),

- Les droits face aux évolutions des techniques ou pratiques médicales (la protection des personnes dans la recherche biomédicale, le respect de l'être humain dès sa naissance; la protection du patrimoine génétique, l'inviolabilité du corps humain ...).

Enfin, il ne faut pas oublier les droits issus des relations entre les administrés et l'Administration comme 22 :

- le droit à l'information administrative,

- le droit à la transparence,

- le droit de participation,

- Etc.

Cette multiplication de l'emploi du terme "droit de, ou de droit à » fait s'interroger sur sa signification réelle. S'agit - il de véritables droits, au sens juridique du terme ou simplement d'une manière plus lisible de définir une politique publique? En effet, un droit n'existe que s'il peut être précisément reconnu en cas de litige devant un juge et si cette juridiction peut prendre des mesures répressives en cas de non-respect. Dans le cas contraire, on est en face d'un objectif à atteindre, un programme à mener et une action publique à entreprendre 23 .

Cette inflation rend utile le tri entre les droits juridiquement opposables et ceux qui ressortent seulement de l'expression juridique d'une politique. Il y a seulement la tentation

21 Kalala Ilunga Mulumba Matthias, Op. cit., p.125.

22 Idem., p.135.

23 Kalala Ilunga Mulumba Matthias, Op. cit., p.135. 
de multiplier des droits comme moyen facile de régler une question difficile. De ce point de vue, cela fait plus penser à un affichage politique opportun qu'à une volonté réelle de régler le problème.

Ce recours facile à la notion de droits de l'homme amène des contradictions avec les droits des précédentes générations. Les droits initiaux correspondent plus à la reconnaissance d'une liberté d'action individuelle. Ils ne connaissent des limites que la liberté d'autrui ou l'intérêt général. Des droits plus récents constituent souvent des droits de créance sur la société. Ils rendent, donc, indispensable une intervention de la puissance publique pour en permettre la réalisation. A l'inverse, une abstention de l'Etat ou des collectivités publiques rend impossible la concrétisation de ces droits.

Certains se demandent, à juste raison, si cette inflation n'est pas en soi préjudiciable, aux (vrais) droits de l'homme et aux libertés fondamentales par un abus de langage, par une forme de tromperie des citoyens. ${ }^{24}$ Cela entraine, il est vrai, une dévalorisation de l'ensemble par une forme de généreuse fuite en avant. Il serait utile parfois de revenir à une juridicité plus assurée et moins factice.

\section{L'ultime protection des droits de l'homme et des libertés fondamentales dans la lutte contre la covid-19}

Affirmer ou proclamer des droits de l'homme et des libertés fondamentales ne suffit pas, il est indispensable de les organiser et de les protéger de manière adaptée au type de danger qu'ils courent. Une bonne organisation et une bonne protection constituent les outils d'une bonne garantie de ces droits et ces libertés. Leur organisation présente quelques spécificités, par rapport à d'autres droits, notamment pour les types de régime juridique mis en œuvre ou le fonctionnement de ces droits ou libertés en périodes exceptionnelles, comme c'est le cas aujourd'hui avec la pandémie à coronavirus-2019.

Les moyens de protection ont été accrus, ces dernières années, aussi bien sur le plan national qu'international. D’une manière générale, on assiste à la généralisation des principes de l'Etat de droit. Lorsqu'on envisage la protection des droits de l'homme et des libertés fondamentales, on est amené à poser plusieurs questions essentielles : une protection contre qui? Une protection par qui? Une protection de quelle manière? ${ }^{25}$

Les droits de l'homme et les libertés fondamentales peuvent être mis en danger aussi bien par les personnes privées que par les autorités publiques. Il s'agit, donc, d'abord d'en protéger l'exercice contre les particuliers selon le précepte que la liberté des uns s'arrête où commence celle des autres. Cela concerne l'ensemble des personnes privées, physiques ou morales. Il s'agit, ensuite, des autorités publiques. Il s'agit, enfin, des politiques que des autorités administratives qui peuvent aussi bien par leur action matérielle que par leur activité juridique porter atteinte aux droits de l'homme et aux libertés. 
Les protecteurs des droits de l'homme et des libertés fondamentales sont divers. Il peut s'agir des juges qui, de manière générale, veillent au respect des lois et, donc, sont des protecteurs naturels de la liberté individuelle. A côté des juges, des nouvelles institutions interviennent afin de compléter la protection des droits de l'homme et des libertés fondamentales.

Les modalités de protection varient, elles prennent des formes juridictionnelles avec des jugements ou des arrêts qui tranchent des litiges ou prennent des sanctions. Elles peuvent aussi prendre des formes non juridictionnelles et plus administratives, ou simplement politiques ou sociétales. Il peut aussi s'agir de démarches répressives et ou préventives.

\section{Les principes directeurs de protections des droits humains dans la lutte contre la covid-19.}

Pour mieux assurer la protection des populations, les nations unies ont mis en place des principes directeurs pour éviter de marcher davantage sur les droits humains. En effet, la maladie à coronavirus 2019 met à l'épreuve les sociétés, les gouvernements, les communautés et les individus. L'heure est à la solidarité et à la coopération afin de lutter contre le virus et d'atténuer les effets, souvent imprévus, des mesures visant à stopper sa propagation. Le respect des droits de l'homme dans tous les domaines, y compris les droits économiques, sociaux, culturels, civils et politiques, sera indispensable pour garantir le succès des mesures de santé publique et se rétablir de cette pandémie ${ }^{26}$.

Ces principes concernent notamment :

- L'accès aux soins de santé,

- Les mesures d'urgence,

- Ne laisser personne de côté,

- Le logement,

- Les personnes handicapées,

- Les personnes âgées,

- Les personnes vivant en détention ou en institution,

- L'information et la participation,

- Etc.

En République Démocratique du Congo, où la fragilité due au flux important des échanges avec les pays affectés l'expose davantage, le pays n'a pas pu échapper à l'importation des cas de covid-19, malgré les mesures de prévention mises en place. Pour faire face à cette situation, le Gouvernement de la République Démocratique du Congo avec l'appui des partenaires n'a pas tardé à recadrer son plan stratégique multisectoriel contre l'épidémie au covid-19.

26 Nations Unies, Droits de l'homme au cour de la riposte, Principes directeurs concernant la covid-19, 08 Mai 2020, p. 1, disponible sur www.ohchr.org. 
Ce plan visait à assurer une réponse efficace dans la ville de Kinshasa et une préparation opérationnelle dans les provinces non encore touchées. Ce plan stratégique aura un ancrage dans le système de santé du pays tout en s'inscrivant aux stratégies de lutte, que propose l'organisation mondiale de la santé, lesquelles sont fonction des phases de l'épidémie, contextualisées selon les réalités du pays ${ }^{27}$.

Les expériences récentes sur les épidémies de la maladie à virus Ebola vont être capitalisées notamment dans la coordination, le laboratoire et recherche, la prise en charge des cas, la prévention et contrôle de l'infection, l'engagement communautaire, la logistique et la surveillance, particulièrement dans le suivi des contacts. Et la mise en place d'une coordination nationale de lutte contre la covid-19 confiée au Professeur Dr Muyembe ${ }^{28}$ n'avait pas attendu.

L'objectif général de ce plan est de contribuer à interrompre la transmission de covid-19 et de minimiser son impact sanitaire et socio-économique sur toute l'étendue de la République Démocratique du Congo pour une période de 12 mois $^{29}$. Tandis que les Objectifs Spécifiques est de renforcer la gouvernance; organiser les structures de prise en charge des malades covid-19; renforcer les capacités de diagnostic de laboratoire; renforcer les capacités de Surveillance et d'investigation des cas; améliorer la prise en charge dans toutes les structures de santé et la communauté; renforcer la communication sur le risque et l'engagement communautaire; renforcer le système logistique d'urgence de covid-19; assurer la Prise en charge Psychosociale; et mettre en œuvre les mesures de mitigations de risque de propagation (mesures de distanciation sociale) ${ }^{30}$.

\section{DES MESURES DE LUTTE CONTRE LA COVID-19 : UN COUP DUR CONTRE LES DROITS HUMAINS EN REPUBLIQUE DEMOCRATIQUE DU CONGO}

\section{Stratégies de lutte contre la covid-19 dans le respect des droits humains.}

Plusieurs stratégies ont été mises en place par les nations unies, les organisations internationales et les gouvernements de différents Etats. Cependant, nous allons présenter ici l'économie de toutes ces stratégies tel que formulées par la note technique du Fonds Mondial. Les menaces que fait peser la pandémie de covid-19 sur les pays sont considérables et multiples, avec des effets potentiellement dévastateurs sur la morbidité et la mortalité pour les personnes, sur les systèmes de santé et sur les programmes financés par le Fonds mondial pour lutter contre le sida, la tuberculose et le paludisme.

27 Plan de préparation et de riposte contre l'épidémie au covid-19 en République Démocratique du Congo, secrétariat technique covid-19, Kinshasa, Mar 2020, p3.

28 C'est encore lui qui avait géré la maladie à virus Ebola; son expérience n'est donc pas à démonter.

29 Plan de préparation et de riposte contre l'épidémie au covid-19 en RD Congo, Op. cit., p.7.

30 Ibidem. 
Par ailleurs, une riposte disproportionnée, discriminatoire ou sans fondement scientifique menace également les droits fondamentaux en matière de santé, en particulier l'accès aux services de santé des communautés les plus marginalisées. Il se peut que d'autres mesures, y compris législatives ou politiques, adoptées par les gouvernements pour endiguer la propagation du covid-19 soient appliquées de telle sorte qu'elles aboutissent à des violations des droits humains (notamment des violences et d'autres mauvais traitements).

En outre, certaines mesures proposées pour des raisons de santé publique pourront ne pas être conformes aux normes internationales en matière de droits humains, notamment du fait de leur caractère inutile, disproportionné ou discriminatoire. Enfin, un diagnostic positif de covid-19 pourra entraîner des phénomènes de rejet dans les familles, les communautés, les foyers et les lieux de travail, même si la personne touchée a complètement recouvré la santé.

Durant cette crise, il faut préconiser une approche fondée sur les droits et soucieuse de l'égalité de genre. Cette détermination permettra au Fonds mondial et aux pays de riposter avec plus d'efficacité à la covid-19 et d'atténuer les éventuelles répercussions négatives de la pandémie sur les programmes actuellement financés par le Fonds ${ }^{31}$.

Pour ce faire, celui-ci entend :

- Porter toute l'attention nécessaire aux populations les plus vulnérables et les plus marginalisées;

- Restreindre les mesures disproportionnées, sans fondement scientifique ou discriminatoires;

- Adapter sa programmation axée sur les droits humains afin d'apporter la meilleure réponse aux défis posés par la pandémie de covid-19;

- Favoriser la participation des communautés à ses processus de programmation, d'élaboration des politiques et de prise de décisions.

L'engagement stratégique du Fonds mondial en faveur des droits humains et de l'égalité de genre vient souligner un principe selon lequel la protection des droits humains va de pair avec la protection de la santé. La période que nous vivons, ni aucune autre situation de crise, ne justifie d'abandonner la protection des droits. Dans la mesure où la pandémie de covid-19 est une menace à la fois pour la santé publique et pour les droits humains, il est plus important que jamais de s'engager dans une riposte fondée sur les droits (et soucieuse de l'égalité de genre).

En plus de s'engager en faveur des droits humains et de l'égalité de genre, le Fonds mondial a également établi cinq normes minimales que tous les maîtres d'œuvre doivent respecter (une condition obligatoire figurant dans tout accord de subvention signé avec le Fonds) :

31 Fonds Mondial, op.cit. pp.2-3. 
- Garantir l'accès aux services à tous sans discrimination, y compris aux personnes en détention;

- Avoir recours exclusivement à des médicaments ou à des pratiques médicales scientifiquement approuvés et éprouvés;

- Ne pas faire appel à des méthodes qui constituent un acte de torture ou s'avèrent cruelles, inhumaines ou dégradantes;

- Respecter et protéger le consentement en connaissance de cause, la confidentialité et le droit au respect de la vie privée concernant le dépistage médical, les traitements ou les services de santé;

- Éviter la détention médicale et l'isolement involontaire, qui ne doivent être utilisés qu'en dernier recours.

\section{Les services publics, garants du droit à la protection de la santé et de la sécurité matérielle de l'individu}

En République Démocratique du Congo, le droit à la santé et à la sécurité alimentaire est garanti. La loi fixe les principes fondamentaux et les règles d'organisation de la santé publique et de la sécurité alimentaire ${ }^{32}$. Le droit à un logement décent, le droit d'accès à l'eau potable et à l'énergie électrique sont garantis. La loi fixe les modalités d'exercice de ces droits ${ }^{33}$. Des nombreux services publics sont ainsi appelés à mettre en œuvre ces exigences dévolues à la protection de la santé ou des moyens d'existence de l'individu.

\section{Les services publics à vocation sanitaire}

Le droit à la santé : les questions de santé sont en permanence au cœur de l'actualité et les sujets qui y mènent particulièrement nombreux : Epidémies, accidents sanitaires, responsabilité médicale, accès aux soins, maitrise de dépenses de santé, progrès de la science, etc. Les exigences sécuritaires étant de plus en plus grandes, il devient impératif de s'interroger sur ce principe du droit à la santé ${ }^{34}$ (droit à la protection de la santé), maintes fois affirmés dans les textes internationaux et afficher au plus haut de la hiérarchie des sources en Droit interne.

La santé occupe et préoccupe ${ }^{35}$ : Qu'est-ce que la santé? La plus célèbre définition est certainement celle de l'Organisation Mondiale de la Santé : la santé n'y est pas seulement définie comme l'absence de maladie ou l'infirmité, mais comme un état de complet bien-être physique, mental, et social. Le droit à la protection de la santé suppose l'accès aux soins, la qualité des soins et leur prise en charge. Sans le droit à la sécurité sociale,

32 Article 47 de la Constitution de la République Démocratique du Congo du 18 Février 2006 telle que modifiée par la loi n¹1/002 du 20 janvier 2011, J.O., Kinshasa, 2006.

33 Article 48 de la Constitution de la RDC.

34 Article 47 de la Constitution de la RDC.

35 Ibidem. 
le principe du droit à la santé est condamné au moins dans sa dimension curative à n'être pas effectif, ou bien à engendrer des inégalités, les soins étant alors réservés à ceux dont la situation économique le permet.

Le droit à la santé supporte mal de contredire cet autre principe constitutionnel qu'est le principe d'égalité ${ }^{36}$. Les inégalités d'accès aux soins pour différentes raisons (frais laissés à charge des patients, localisation géographique des offres de soins...) sont pourtant nombreuses. Le droit fondamental à la protection de la santé comprend la prévention, la garantie de l'égal accès aux soins, l'assurance de la continuité des soins et la meilleure sécurité sanitaire possible, mais aussi : le droit de chaque personne malade au respect de sa dignité, l'interdiction des discriminations dans l'accès à la prévention et aux soins, le droit de chaque personne au secret des informations la concernant, etc.

A cela s'ajoutent les conditions du consentement libre et éclairé à l'acte médical, le droit à l'information et à la transparence thérapeutique, l'accès au dossier médical, etc. ${ }^{37} \mathrm{La}$ réalisation des droits sociaux suppose souvent une action plus intense de l'Etat : technique de la réglementation et technique du service public (l'Etat prend alors en charge lui-même la satisfaction des besoins) sont alors mises à contribution. Certes, dans la lutte contre la covid-19, toute la prophylaxie est faite aux dépens de l'Etat congolais, mais il faut signaler les multiples difficultés qui ont toujours caractérisé le système sanitaire congolais en situation normale.

Assurer les conditions d'existence de l'individu est une visée large et ambitieuse; mais, qui dissimule, dans son ampleur même, certaines implications évidentes. Aucun « droitcréance » ne conditionne ainsi plus fondamentalement le développement des citoyens que la protection de la santé. Or cette exigence constitutionnelle ${ }^{38}$ nécessite l'instauration d'un service public dédié à sa mise en œuvre. La généralité des destinataires de cette exigence implique, en effet, une prise en charge de cette activité par les pouvoirs publics, seuls à même de mettre en œuvre un égal accès aux soins la garantissant concrètement sur l'ensemble du territoire et à tout instant.

Cette affirmation ne saurait, cependant, tendre à remettre en cause l'existence d'un secteur médical privé subvenant, dans la pratique, à une grande partie des besoins de la population en matière de soins. Le remède est constitué par l'instauration d'un service public de santé, seul susceptible de garantir que chacun puisse être soigné, dans le cadre d'un système de "complémentarité-concurrence » avec le secteur privé. La satisfaction du droit à la protection de la santé suppose ainsi obligatoirement la mise en place et le maintien d'un service public constitutionnel de santé coexistant avec un secteur privé, la distinction public/privé tendant aujourd'hui à s'effacer au sein d'un système de santé envisagé de manière globale.

36 Articles 11-13 de la Constitution de la RDC.

37 Kalala Ilunga Mulumba Matthias, op.cit., p.263.

38 CAMBRILLAC, R., et autres, Op.cit., p.800. 
En pratique, la garantie de cette exigence se matérialise par l'intervention de multiples services publics à vocation sanitaire. Les établissements publics de santé, centres hospitaliers et hôpitaux locaux, assurent le service public hospitalier, lequel comprend « les examens de diagnostic, la surveillance et le traitement des malades, des blessés et des femmes enceintes ", mais également l'enseignement universitaire et la recherche, la formation continue des praticiens hospitaliers, les actions de médecine préventive et d'éducation pour la santé, l'aide médicale urgente et la lutte contre l'exclusion sociale ${ }^{39}$.

Ces établissements publics demeurent souvent formellement rattachés à une collectivité locale; mais, restent pilotés par l'Etat à travers les agences régionales de l'hospitalisation, groupement d'intérêt public chargé de définir et de mettre en œuvre la politique de soins hospitaliers. En République Démocratique du Congo, le tableau sombre des conditions dans lesquelles preste le personnel de santé nous invite à rendre hommage aux ministères de la santé publique et de l'Enseignement Supérieur et Universitaire, à travers le travail remarquable que nos jeunes médecins réalisent avec beaucoup de sacrifices dans les conditions quasi inacceptables ${ }^{40}$.

En effet, d'une part dans la plupart des Zones de santé et Hôpitaux de la République Démocratique du Congo, les équipements, l'eau et l'électricité font défaut, d'une part l'environnement est favorable à la transmission des maladies nosocomiales : conditions hygiéniques calamiteuses, toilettes de fortune dégageant des odeurs nauséabondes, blocs opératoires et appareils d'analyse inexistant, nombreuses opérations nocturnes faites à l'aide de l'éclairage de la bougie, du téléphone portable ou de la lampe tempête, lits de malades en nombre très insuffisant et sans garnitures, ruptures récurrentes de stocks des produit pharmaceutiques, toitures de maisons d'habitation de médecins et certains hôpitaux et centre de santé en paille, etc. ${ }^{41}$. En dépit de ce spectacle désolant, des multiples témoignages concordants en provenance des milieux ruraux confirment que plusieurs vies humaines sont sauvées grâce à la présence de nos jeunes médecins ${ }^{42}$.

\section{Les services publics protégeant l'individu face à certains risques de l'existence}

Les droits sociaux du citoyen : notons à priori que la société laisse le citoyen gagner sa vie sans assistance. Toutes fois, elle doit lui garantir et garantir à la famille un droit au minimum. Elle doit aider, en outre, le citoyen à trouver l'activité économique qui lui permettra de subvenir à ses besoins. Le droit au minimum suppose : l'aide sociale à l'enfance ${ }^{43}$; l'allocation de solidarité; l'allocation aux adultes handicapés ${ }^{44}$; l'allocation de

39 CAMBRILLAC, R., et autres, Op.cit., p.800.

40 Kalala Ilunga Mulumba Matthias, op.cit., p.264.

41 Actes majeurs du symposium organisés par l’Université de Kamina, juin 2015, inédit.

42 Ibidem.

43 Articles 41 de la Constitution de la RDC.

44 Articles 49 de la Constitution de la RDC. 
solidarité aux personnes âgées ${ }^{45}$; les allocations familiales et l'aide sociale aux familles. Il faut malheureusement noter que ces droits ne sont souvent pas respectés dans la plupart des pays en développement, en général, et en République Démocratique du Congo, en particulier.

Les droits économiques du citoyen, nous pensons ici aux : droit à l'instruction ${ }^{46}$, droit à la formation professionnelle ${ }^{47}$; droit à l'emploi ${ }^{48}$ et; devoir de travailler ${ }^{49}$. La Nation garantit également la « sécurité matérielle », c'est-à-dire fournir à l'individu une assurance contre certains risques. Il n'est alors plus directement question de protection de la santé, attachée en priorité à une activité de diagnostic et de soin; mais, d'une protection économique des individus contre certaines difficultés. Les deux domaines restent, cependant, très liés et leur distinction, si elle offre une meilleure lisibilité, se révèle parfois artificielle, « l'action sociale impliquant la prise en compte de la dimension sanitaire, l'action en faveur de la santé devenant tantôt une condition, tantôt une composante de la politique sociale ».

3. Analyse-critique des mesures prises par les autorités congolaises dans la lutte contre la covid-19

Touchée par la pandémie en mars 2020, la République Démocratique du Congo avait, à l'instar de tous les Etats du monde, pris toutes les mesures idoines pour prévenir et prendre en charge toutes les personnes touchées par le coronavirus 2019. Cependant, ces mesures ont, au dire de la plupart des interviewées ${ }^{50}$, brillées par des violations massives des droits humains. En voici brièvement une illustration :

a) Imposition de la distanciation sociale : cette mesure n'a jamais été scrupuleusement observée. En effet, on a vu et voit encore des marches de soutien ou de protestation s'effectuer en toute aisance, comme si on était en période normale, des attroupements des individus un peu partout sur l'ensemble du territoire national.

b) Confinement (partiel ou total) : A Kinshasa, Lubumbashi, Likasi, Kasumbalesa, il y a été organisé des confinements, mais ceux-ci ont seulement été une simple occasion de violation des droits humains. Comment s'évertuer à confiner totalement une population qui ne vit que de l'économie informelle, de la débrouillardise sans aucune mesure d'accompagnement en termes d'allocation?

c) Restrictions sur les rassemblements sociaux / publics : malgré cette interdiction, on a assisté à des spectacles désolants des marches de soutien ou de protestation des partis politiques et ou regroupements politiques en pleine période de confinement (Union

45 Ibidem.

46 Articles 43 de la Constitution de la RDC.

47 Ibidem.

48 Articles 36 de la Constitution de la RDC.

49 Ibidem.

50 Pour des raisons de sécurité et ou personnelles, la plupart d'interviewés ont requis l'anonymat. 
pour la démocratie et le progrès social parti au pouvoir, le Front commun pour le Congo avec en tête le Parti du peuple pour la reconstruction et le développement, Solidarité congolaise pour la démocratie, Union nationale des fédéralistes du Congo, etc.). En outre, les marchés n’ont jamais été fermés et constituent des véritables lieux de rassemblements pouvant favoriser la circulation aisée du virus.

d) Les transports en commun : des arrêtés des autorités politico-administratives avaient été pris pour limiter le nombre des passagers dans les transports en commun, mais cela n'a jamais été observé dans toutes les villes de la République Démocratique du Congo.

e) Désinfection des espaces publics : encore un autre moment pour le personnel commis à cette tâche de se livrer au vol et détournement des fonds y relatifs.

f) Fermeture de toutes les structures scolaires, universitaires et de formation : plus d'un interviewé ne voit pas l'importance de les fermer. Dès lors que la mesure de distanciation n'est pas observée, pourquoi les fermer. Il s'agit là d'une imitation pure et simple et qui n'a rien à voir avec les réalités congolaises et qui viole le droit à l'éducation. D'ailleurs, beaucoup de voix se sont levées pour demander la réouverture sans condition mais sans succès.

g) Fermeture des restaurants, bars et discothèques : cette mesure n'avait non plus jamais été respectée, la plupart de bars et restaurants avaient fermés seulement les entrées de devant alors que les activités se déroulaient normalement par le derrière, la corruption y aidant. Seuls les récalcitrants qui ne donnaient pas de l'argent pouvaient être torturés et dépouillés de leurs téléphones et autres bien de valeurs.

h) Encouragement à une hygiène rigoureuse (par exemple, se laver fréquemment les mains, etc.) : cette mesure est restée jusqu'à ce jour une simple formalité. On voit dans tous les bureaux des différentes administrations, des fontaines, lave-mains qui ne servent que des simples ornements, du formalisme pur et simple. Cependant, certaines institutions comme les banques et l'Eglise Catholique sont intraitables dans le respect de cette mesure.

i) Fermeture des frontières (terrestres, aériennes, maritimes) : des cas de fraudes étaient également signalés toujours grâce à la corruption.

j) Couvre-feux : aux approches des festivités marquant la fin de l'année jusqu'à ce moment où nous rédigeons le présent article, il a été décidé un couvre-feu de 21h00-5h00 : sur terrain qu'est-ce qu'on observe? Cette opération est une véritable occasion pour les hommes en uniformes, qui souvent commencent plutôt que prévu, de se livrer aux vols, viols, extorsions, tortures et violences de tout genre ${ }^{51}$.

k) Port obligatoire du cache-nez : une mesure aussi fantaisiste que la plupart des précédentes en République Démocratique du Congo. En effet, là où cette mesure est appliquée rigoureusement comme dans certaines banques ou dans l'église Catholique, au lieu d'être un cache-nez, celui-ci devient simplement cache-menton. C'est-à-dire, on le met uniquement par pur formalisme pour entrer, une fois admis, il est vite totalement ou

51 Propos recueillis auprès de plusieurs personnes qui ont toutes requis l'anonymat. 
partiellement enlevé. Parfois, pour ceux qui utilisent les cache-nez médicaux, on note le non-respect du temps de son utilisation, parfois, certains utilisateurs se permettent même le réemploi.

\section{CONCLUSION}

Eu égard à ce tableau sombre que nous venons de présenter dans l'application des mesures de lutte contre la covid-19, il y a lieu d'affirmer sans crainte d'être contredit que la violation des droits humains se porte bien en République Démocratique du Congo. Ainsi, sans méconnaître les efforts, combien louables des autorités congolaises, il est fort triste de constater que la lutte contre la pandémie à coronavirus 2019 est un véritable coup dur porté contre les droits humains qui, même en temps normal, y ont toujours fait l'objet de multiples violations.

Des enseignements méritent donc d'être tirés de cette situation de covid-19 pour rendre les services publics congolais efficaces, efficients, émergents et compétitifs de façon à leur permettre de faire face à toutes autres éventualités. Il s'agira donc, de se demander dans les jours à venir comment, en fonction de ce qui se passe, améliorer la capacité des services publics congolais à affronter les crises futures qui, par définition, ne ressembleraient pas nécessairement à la crise de covid-19. Chaque épisode apportant son lot d'inconnues qui conduirait à gérer des situations inédites, les gouvernants de la République Démocratique du Congo devraient désormais savoir anticiper et réagir à bon escient.

\section{BIBLIOGRAPHIE}

\section{TEXTES DE LOIS}

1. Déclarations Universelles des Droits de l'Homme du 10/12/1948

2. Charte Africaine des Droits de l'Homme et des peuples

3. Charte Africaine sur les valeurs et les principes du service public et de l'Administration

4. Constitution de la République Démocratique du Congo du 18 Février 2006 telle que modifiée par la loi n¹1/002 du 20 janvier 2011, J.O., Kinshasa, 2006.

\section{OUVRAGES ET AUTRES DOCUMENTS}

1. Actes majeurs du symposium organisés par l'Université de Kamina, juin 2015, inédit.

2. Amnisty International, mesures prises face à la covid-19 et obligations des états en matière de droits humains : observations préliminaires, Déclaration publique du 12/03/2020, disponible sur www.amnesty.org

3. BONNARD, R., Précis de Droit administratif, $3^{\text {ème }}$ éd., LGDJ, Paris, 1940.

4. CABRILLAC (Rémy), FRISON-ROCHE (Marie-Anne) et REVET (Thierry), Libertés et droits fondamentaux, Dalloz, Paris, 14ème éd., 2008. 
5. Fons mondial, Note d'orientation : les droits humains en temps de pandémie de COVID-19, 14 avril 2020.

6. Guglielmi, Gilles J., introduction au droit des services publics, éd. Dalloz, Paris, 1993.

7. JEZE, G., Principes généraux du Droit Administratif, 3è éd. Tome I, Giard, 1970.

8. Kalala Ilunga Mulumba Matthias, Aspects et mécanismes de contrôle de la satisfaction de l'intérêt général en République Démocratique du Congo, Mémoire de DEA, Université de Lubumbashi, 2015, inédit.

9. Kalala Ilunga Mulumba Matthias, Gestion des services publics et protection des droits humains, thèse de doctorat, Université de Lubumbashi, 2019, inédit.

10. LACHAUME, J.F., BOITEAU, \& PAULIAT, H., Droit des services publics, éd. $3^{\text {ème éd. }}$ Coll. Armand Colin, éd. Dalloz, Paris, 2004.

11. LE MASNE, P., Services publics et développement, GEDES, Université de Poitiers.

12. Les droits de l'homme au cœur de la riposte, principes directeurs concernant la covid-19, 8 mai 2020 disponible sur www.ohchr.org consulté le mardi le 15/12/2020.

13. MORANGE, J., Libertés publiques, éd. PUF, Paris 2007.

14. MOURGEON, J., Les droits de l'homme, coll. Que sais-je?, 8éme éd. PUF, Paris, 2003

15. OBERDORFF Henri, Droits de l'homme et libertés fondamentales, L.G.D.J., Paris, 2008.

16. OBERDORFF Henri, Droits de l'homme et libertés fondamentales, L.G.D.J., Paris, 2008

17. OMS, "WHO characterizes COVID-19 as a pandemic", 11 mars 2020, disponible sur www.who.int/emergencies/diseases/novel-coronavirus-2019/events-as-they-happen.

18. Pierre Villeneuve, L'ordre public sanitaire, JCP / la semaine juridique - édition administrations et collectivités territoriales $N^{\circ}$ 12.23 Mars 2020 - (C) LEXISNEXIS SA.

19. République Démocratique du Congo, Gouvernement, Plan de préparation et de riposte contre l'épidémie au covid-19 en République Démocratique du Congo, secrétariat technique covid-19, Kinshasa, Mar 2020.

20. PNUD (2003), Rapport mondial sur le développement humain 2003, Economica.

21. Rapport analytique du webinaire, 23 avril 2020, l'impact de la crise du Covid-19 sur le constitutionnalisme et l'État de droit dans les pays francophones et lusophones d'Afrique de l'Ouest et d'Afrique Centrale, Institute international pour la démocratie et l'assistance électorale, disponible sur http://www.idea.int consulté le 15/12/2020

22. République Démocratique du Congo, Gouvernement, Impacts sanitaires et socio-économiques de la covid-19 en République Démocratique du Congo, analyse prospective et orientations de la riposte multisectorielle, Kinshasa, mai 2020.

23. VUNDUAWE Te Pemeko, F., Traité de droit administratif, Ed. Larcier (Afrique édition), Bruxelles, 2007. 